\title{
A game-theoretic learning approach to QoE-driven resource allocation scheme in 5G-enabled loT
}

\author{
Haibo Dai ${ }^{1}$, Haiyang Zhang ${ }^{2}$, Wei $\mathrm{Wu}^{3}$ and Baoyun Wang ${ }^{3 *}$
}

\begin{abstract}
To significantly promote Internet of Things (IoT) development, $5 \mathrm{G}$ network is enabled for supporting loT communications without the limitation of distance and location. This paper investigates the channel allocation problem for loT uplink communications in the $5 \mathrm{G}$ network, with the aim of improving the quality of experience (QoE) of smart objects (SOs). To begin with, we define a mean opinion score (MOS) function of transmission delay to measure QoE of each SO. For the sum-MOS maximization problem, we leverage a game-theoretic learning approach to solve it. Specifically, the original optimization problem is equivalently transformed into a tractable form. Then, we formulate the converted problem as a game-theoretical framework and define a potential function which has a near-optimum as the optimization objective. To optimize the potential function, a distributed channel allocation algorithm is proposed to converge to the best Nash equilibrium solution which is the global optimum of maximizing the potential function. Finally, numerical results verify the effectiveness of the proposed scheme.
\end{abstract}

Keywords: Heterogeneous network, loT, MOS, Resource allocation, Potential game

\section{Introduction}

The Internet of Things (IoT) is a system of human-toobject or object-to-object connection that sensors, controller, mechanical and digital machines, objects, animals, or people are interrelated and transfer data over a network by using information technology $[1,2]$. In IoT, a thing can be a person with wearable devices, an autonomous vehicle with sensors, a farm animal with biochip transponders, or any other smart objects (SOs) provided with the ability to transfer data over a network [3, 4]. The concept of IoT is first mentioned by Kevin Ashton in a presentation he made to Procter Gamble in 1999. At that time, the computers use the data they gathered with the help from human beings [5]. However, people have very limited time, attention, and accuracy, which results in that they are not very good at capturing data about things in reality. The enormous potential demand for things connection drive the rapid development of IoT. IoT SOs contain

\footnotetext{
*Correspondence: bywang@njupt.edu.cn

${ }^{3}$ College of Telecommunications and Information Engineering, Nanjing University of Posts and Telecommunications, 66 Xin mofan Road, 210003 Nanjing, China

Full list of author information is available at the end of the article
}

different types, in which some are sensitive for delay, some are need for high reliability, and some are low-power and low-cost. Moreover, most of the IoT traffic is in the uplink and IoTs' messages are typically small in size and sparse in time. These characteristics of IoT SOs make their access to the network different from classical users, which brings the network a great challenge [6]. Therefore, providing satisfactory service for IoT applications with differentiated demands is an important field, and the requirement for ultra-reliable low-latency communications of IoT SOs is greatly emphasized.

5G heterogeneous networks are envisioned to play a key role in providing a promising infrastructure for the massive proliferation of IoT SOs and the corresponding services [7-11]. IoT SOs with very limited computing and storage capabilities are associated with access points of 5G network for cloud services and communications $[12,13]$. To handle the massive connectivity and satisfy the requirements of ultra-reliable low-latency communications, 5G network supporting IoT communications requires huge spectrum resources or the improvement of spectral efficiency [14]. Moreover, the interference management problem is one of the key challenges in 
5G-enabled IoT, since the co-channel model that small cell base stations (SCBSs) overlayed on the covering area of macrocell base station (MBS) share the same frequency band is generally adopted in 5G network [15]. The resource allocation strategies are usually optimized to overcome the interference problem [16]. In particular, the requirement of ultra-reliable low-latency communications for IoT SOs is greatly emphasized, and thus, the performance enhancement of quality of experience $(\mathrm{Q} o \mathrm{E})$ of IoT is a challenging and attractive research area. Motivated by achieving a real-time and reliable transmission of IoT, a QoE-driven resource allocation scheme is proposed in this paper.

Next, we give a brief review of the works related to our research. More related works on the efficient IoT support in 5G can be found in [17-19]. Yerrapragada and Kelley [17] investigated a perfect interference alignment scheme for multiple-input multiple-output systems and applied it to a 5G-enabled IoT architecture. Since intercell interference significantly degraded the performance of IoT communications, Dao et al. [18] proposed a novel algorithm for finding the most appropriate pair of IoT terminal or its associated BS to provide a relay-assisted communication for the IoT terminal with poor signals in the inter-cell interference area. For IoT in cognitive 5G networks, the multiband cooperative spectrum sensing and resource allocation framework was presented in [19]. IoT communications in 5G network are expected to provide flexible delivery of broad services with a high QoE. Recently, the research on improving QoE of IoT SOs has attracted more and more attention [20-22]. Aminjavaheri et al. [20] presented an underlay control signaling method for ultra-reliable low-latency communication applications in an LTE network and analyzed its performance. Since the satisfaction of QoE becomes the major challenge in content-centric IoT, the authors in [21] have analyzed lots of factors, i.e., content popularity and weight factor, which impact the resource allocation and how they subsequently influence the QoE. As a cloud resource, fog computing is rationally used for the delay-sensitive services of IoT by minimizing resource underutilization and enhancing QoE [22].

Resource allocation in IoT is investigated in many literatures by using game theory [23-27]. Huang et al. [23] employed a cooperative game to model and analyze device-to-device communication for achieving highperformance data transportation in the new cloudcentric IoT paradigm. Device-to-device communication underlaying cellular networks was investigated in [24] to improve spectral efficiency, and a game-theoretic resource allocation scheme was designed by exploring the inherent competition of spectrum resource among users. The authors in [25] proposed Stackelberg game and manyto-many matching to solve the multi-stage problems of pairing, resource pricing, and purchasing in three-tier IoT fog networks. Although the proposed framework can achieve high performance, the optimal solution is ambiguous. In addition, matching theory was also used in [26] to find a stable IoT node pairing. In [27], the problem of efficiently and effectively securing IoT networks was investigated by carefully allocating security tools.

\section{Methodology}

Bearing the above in mind, we tend to leverage the gametheoretic learning algorithm to solve the resource allocation problem in 5G-enabled IoT network. In this paper, we assume that there are some SOs that access to $5 \mathrm{G}$ network, and it is looking forward to achieving the effective deployment of IoT without considering the limitation of distance and location. Certainly, this is confronted with more challenges. SOs are usually sensitive to latency, which raises a higher demand for data transmission. However, the interference, deriving from the reuse of radio resource, greatly affects SOs' QoE in 5G network. The non-convex and integer optimization objective brings a great challenge to achieve the rational allocation of resource. Moreover, a distributed algorithm is desired for various SOs with different service requirements. In this paper, we study the channel allocation problem by applying game theory to analyze the distributed decisions made by SOs, and perform the learning algorithm to maximize the sum-MOS of SOs in the 5G network. The main contributions of our work are summarized as follows:

- We consider the QoE of all SOs in the 5G-enabled IoT network as the objective function. A MOS standard in terms of the data transmission delay is proposed to measure QoE of various services. Then, an equivalent form is derived to replace the original optimization objective.

- We use the game-theoretic model to formulate the modified optimization problem in which the designed potential function is an approximation of the optimization objective. Then, we prove it to be an exact potential game, whose best Nash equilibrium (NE) point is a near-optimal solution of the original optimization problem.

- To find the best NE point, we design a distributed learning algorithm which can asymptotically converge to the global optimal solution that maximizes potential function with arbitrary high probability.

The rest of this paper is organized as follows. In Section 3, the system model and the QoE metric are presented. Then, the proposed channel allocation problem is equivalently converted into a tractable problem. According to the converted optimization problem, Section 4 establishes a game framework and then investigates the 
properties of the equilibrium. In Section 5, we propose an algorithm and the asymptotical optimality is verified. Finally, numerical results and discussion are presented in Section 6, and Section 7 concludes this paper.

\section{System model and problem formulation}

\subsection{System model}

We consider an uplink 5G-enabled IoT network consisting of $B$ BSs, $K$ diverse SOs (i.e., smart phone, smart meter, wearable device, and monitoring device) and $N$ orthogonal channels, illustrated by Fig. 1. The set of all BSs is denoted by $\mathcal{B}=\{1,2, \ldots, B\}$ and the set of all SOs is represented by $\mathcal{K}=\{1,2, \ldots, K\}$. Suppose that the associations between BSs and SOs have been predetermined and let $b_{k} \in \mathcal{B}$ be the BS at the service of SO $k \in \mathcal{K}$. Moreover, suppose that each SO chooses a channel for data transmission and the bandwidth of each orthogonal channel is the same. We denote the set of the channels by $\mathcal{N}=\{1,2, \ldots, N\}$. Let $a_{k}$ be the channel allocation strategy of SO $k \in \mathcal{K}$ and $\mathcal{A}_{k}$ be the set of all possible selections for $k$. Thus, $\mathbf{a}=\left(a_{1}, a_{2}, \ldots, a_{K}\right)$ is the channel selection profile for all SOs and $\mathcal{A}=\mathcal{A}_{1} \times \mathcal{A}_{2} \times \cdots \times \mathcal{A}_{K}$ is the space of all possible selections for all SOs.

The channel from $\mathrm{SO} k \in \mathcal{K}$ to $\mathrm{BS} b_{k} \in \mathcal{B}$ is supposed to be flat fading and the channel gain is denoted by $h_{k, b_{k}}$. Let $p_{k}$ be the transmit power of SO $k$. Then, the received signal-to-interference-plus-noise ratio (SINR) of $\mathrm{BS} b_{k}$ from $\mathrm{SO} k$ is given by:

$$
\gamma_{k}=\frac{p_{k} h_{k, b_{k}}}{\sum_{l \in \mathcal{K} \backslash\{k\}} p_{l} h_{l, b_{k}} \mathbb{1}_{\left\{a_{l}=a_{k}\right\}}+\sigma_{k}^{2}},
$$

where $\sigma_{k}^{2}$ is the power of the additive white Gaussian noise at the $\mathrm{BS}$ associated by SO $k$, and the indicator variable $\mathbb{1}_{\left\{a_{l}=a_{k}\right\}} \in\{0,1\}$ is used to denote that the channel allocated to SO $k$ is occupied by SO $l$, i.e., $\mathbb{1}_{\left\{a_{l}=a_{k}\right\}}=1$, or not occupied, i.e., $\mathbb{1}_{\left\{a_{l}=a_{k}\right\}}=0$.

In IoT, different SOs perform different applications, i.e., picture/video collection, game, file upload, and control information transmission. When performing different applications, SOs need to transfer different sizes of data for purpose of obtaining the same user experience in the same period of time. Mathematically, the set of service types required by $S O s$ is represented as $\mathcal{S}=\{1,2, \ldots, S\}$, and $s_{k} \in \mathcal{S}$ is denoted as the performed service type of $\mathrm{SO}$ $k$. Let $C_{s_{k}}$ be the amount of data required from SO $k$ during a given period of time. Hence, the uplink transmission time from $\mathrm{SO} k$ is described as follows.

$$
T_{k}=\frac{C_{s_{k}}}{R_{k}}
$$

where $R_{k}=B \log _{2}\left(1+\gamma_{k}\right)$ is the achievable rate of $\mathrm{BS} b_{k}$ from SO $k$ and $B$ is the bandwidth of each channel.

\subsection{QoE metric}

To measure QoE of various services, we propose a mean opinion score (MOS) standard, ranging from 1 to 5 , in terms of the data transmission delay. Letting $\tau_{1, s_{k}}$ and $\tau_{2, s_{k}}$ be respectively the most satisfied delay and the maximal tolerable delay based on the different service types, the MOS is defined as follows.

$$
\operatorname{MOS}_{k}(\mathbf{a})=\left\{\begin{array}{l}
5, T_{k} \leq \tau_{1, s_{k}}, \\
\alpha \ln \frac{\tau_{1, s_{k}}+\tau_{2, s_{k}}-T_{k}}{\beta}, \tau_{1, s_{k}}<T_{k}<\tau_{2, s_{k}}, \\
1, T_{k} \geq \tau_{2, s_{k}},
\end{array}\right.
$$

where $\alpha=\frac{4}{\ln \tau_{2, s_{k}}-\ln \tau_{1, s_{k}}}$ and $\beta=\tau_{1, s_{k}}\left(\frac{\tau_{1, s_{k}}}{\tau_{2, s_{k}}}\right)^{\frac{1}{4}}$. Figure 2 shows the curve variation tendency of MOS with the

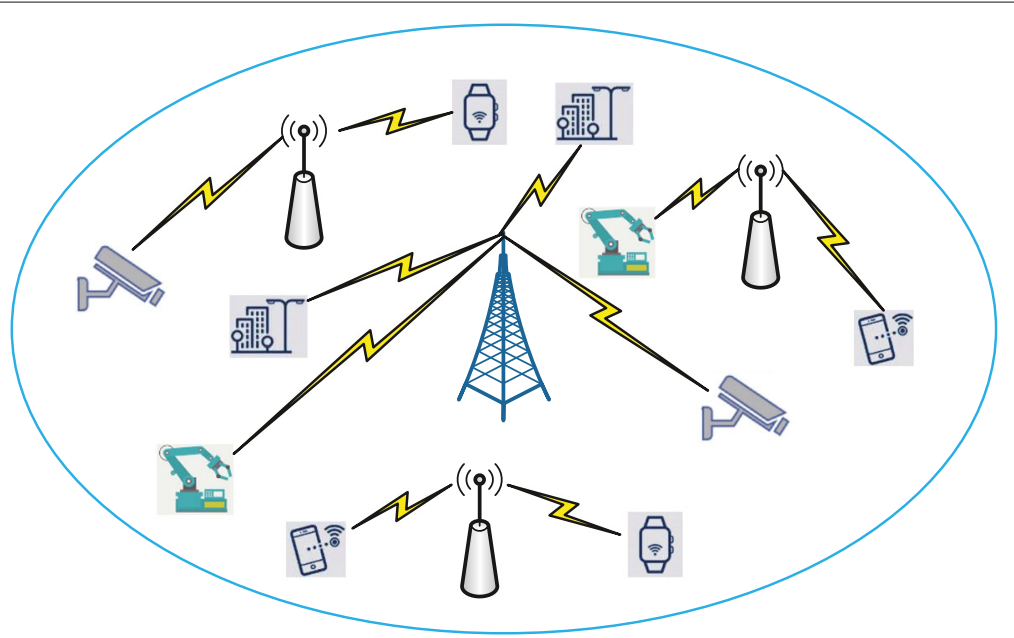

Fig. 1 The 5G-enabled loT network 


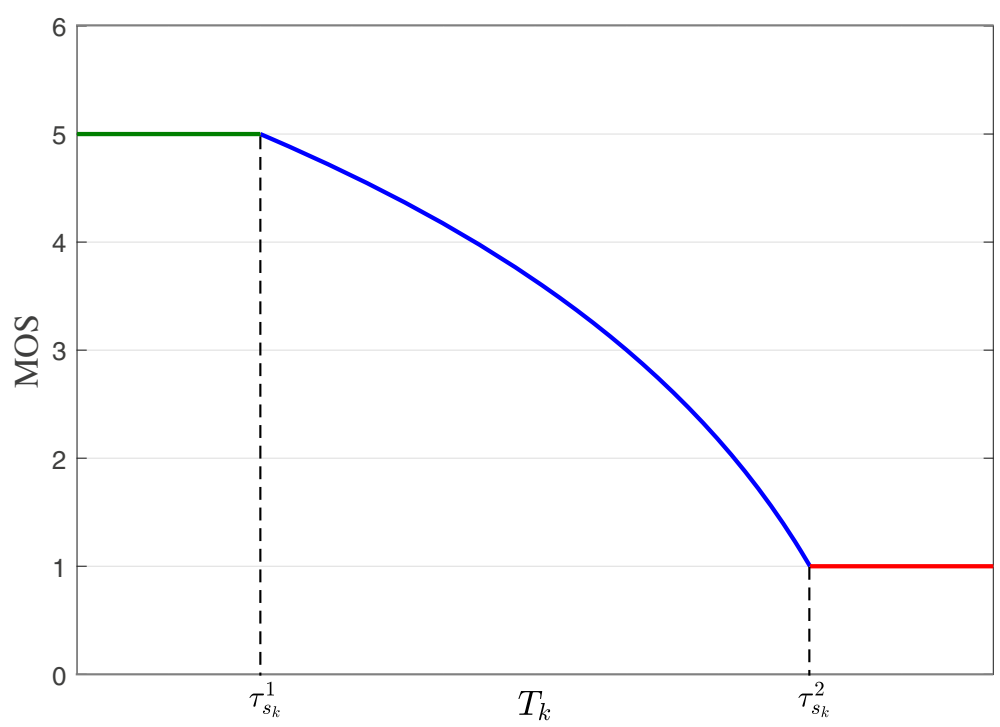

Fig. 2 The QoE metric

change of delay. The MOS values range from 1 to 5 , where MOS $=1$ represents an unacceptable QoE for SOs and MOS $=5$ reflects an excellent user experience. In general, SOs have different tolerances for delay with regard to the different services. The application characteristics of SOs are determined by $\tau_{1, s_{k}}$ and $\tau_{2, s_{k}}$.

\subsection{Problem formulation and transformation}

In this paper, to improve the overall transmission performance of the 5G-enabled IoT network by the optimization of channel allocation, we consider the sum-MOS maximization problem, which is mathematically expressed as:

$$
P: \max _{\mathbf{a} \in \mathcal{A}} \sum_{k \in \mathcal{K}} \operatorname{MOS}_{k}(\mathbf{a})
$$

The problem $P$ is a non-convex and discrete optimization problem, for which finding its solution is expected to be very challenging. In what follows, We convert it into a tractable form. For notational convenience, we first define $U_{k}=p_{k} g_{k, b_{k}}$ and $I_{k}\left(a_{k}, \mathbf{a}_{-k}\right)=\sum_{l \in \mathcal{K} \backslash\{k\}} p_{l} g_{l, b_{k}} \mathbb{1}_{\left\{a_{l}=a_{k}\right\}}+\sigma_{k}^{2}$, where $\mathbf{a}_{-k}$ is the channel selection profile of all the SOs except $\mathrm{SO} k$. Then, we have

$$
T_{k}(\mathbf{a})=\frac{C_{s_{k}}}{B \log _{2}\left(1+\frac{U_{k}}{I_{k}(\mathbf{a})}\right)} .
$$

By using first-order approximation of Taylor expansion at the point $\mathbf{a}^{\prime}, T_{k}$ is expanded as $\tilde{T}_{k}$, namely,

$$
\begin{aligned}
\tilde{T}_{k}\left(\mathbf{a}^{\prime}, \mathbf{a}\right) & \triangleq T_{k}\left(\mathbf{a}^{\prime}\right)+\frac{\mathrm{d} T_{k}\left(\mathbf{a}^{\prime}\right)}{\mathrm{d} I_{k}}\left(I_{k}(\mathbf{a})-I_{k}\left(\mathbf{a}^{\prime}\right)\right) \\
& =\Delta_{1, k} I_{k}(\mathbf{a})+\Delta_{2, k},
\end{aligned}
$$

where $\Delta_{1, k}=\frac{B C_{s_{k}} U_{k}}{\ln 2 T_{k}^{2}\left(\mathbf{a}^{\prime}\right)\left(I_{k}^{2}\left(\mathbf{a}^{\prime}\right)+U_{k} I_{k}\left(\mathbf{a}^{\prime}\right)\right)}$ and $\Delta_{2, k}=$ $T_{k}\left(\mathbf{a}^{\prime}\right)-\Delta_{1, k} I_{k}\left(\mathbf{a}^{\prime}\right)$.

According to (6), (3) is expanded at the point a', namely,

$$
\begin{aligned}
& \widetilde{\operatorname{MOS}}_{k}\left(\mathbf{a}^{\prime}, \mathbf{a}\right) \\
& =\left\{\begin{array}{l}
5, I_{k}(\mathbf{a}) \leq \tilde{\tau}_{1, s_{k}}, \\
\Delta_{3, k} I_{k}(\mathbf{a})+\Delta_{4, k}, \tilde{\tau}_{1, s_{k}}<I_{k}(\mathbf{a})<\tilde{\tau}_{2, s_{k}}, \\
1, I_{k}(\mathbf{a}) \geq \tilde{\tau}_{2, s_{k}},
\end{array}\right.
\end{aligned}
$$

where $\tilde{\tau}_{1, s_{k}}=\frac{\tau_{1, s_{k}}-\Delta_{2, k}\left(\mathbf{a}^{\prime}\right)}{\Delta_{1, k}\left(\mathbf{a}^{\prime}\right)}, \quad \tilde{\tau}_{2, s_{k}}=\frac{\tau_{2, s_{k}}-\Delta_{2, k}\left(\mathbf{a}^{\prime}\right)}{\Delta_{1, k}\left(\mathbf{a}^{\prime}\right)}$, $\Delta_{4, k}=\alpha \ln \frac{\tau_{1, s_{k}}+\tau_{2, s_{k}}-T_{k}\left(\mathbf{a}^{\prime}\right)}{\beta}-\Delta_{3, k} I_{k}\left(\mathbf{a}^{\prime}\right)$, and $\Delta_{3, k}=$ $\frac{\alpha \beta}{T_{k}\left(\mathbf{a}^{\prime}\right)-\left(\tau_{1, s_{k}}+\tau_{2, s_{k}}\right)}$.

By comparing (6) with (3), it is noted that (4) and $\sum_{k \in \mathcal{K}} \widetilde{\operatorname{MOS}}_{k}\left(\mathbf{a}^{\prime}, \mathbf{a}\right)$ have the same solution when $\mathbf{a}^{\prime}=\mathbf{a}^{*}$ where $\mathbf{a}^{*}$ is the optimal solution to (4). Therefore, the original problem (4) is equivalently transformed into the following optimization problem.

$$
\tilde{P}: \max _{\mathbf{a}^{\prime}=\mathbf{a}^{*}, \mathbf{a} \in \mathcal{A}} \sum_{k \in \mathcal{K}} \widetilde{\operatorname{MOS}}_{k}\left(\mathbf{a}^{\prime}, \mathbf{a}\right) .
$$

According to the above definition, it is noted that MOS of each SO depends on not only its channel selection strategy, but also on other SOs' strategies. If too many SOs occupy the same channel to transmit data, the transmission rates are relatively low, and then the low MOSs lead to low-efficient data processing and put pressure on the data storage. Due to the interdependent and interactional relationship among different SOs, we adopt game theory 
to model and analyze the channel allocation strategies of SOs in $\tilde{P}$. Furthermore, it is difficult for each $\mathrm{SO}$ to obtain other information of SOs with different types, which motivates us to propose a distributed learning algorithm for achieving the equilibrium solution of the game modeled from the channel access problem.

\section{Game-theoretic analysis}

In this section, we study the distributed optimization of the channel access problem by using game theory. Every $\mathrm{SO}$ is regarded as a player in the game, and the channel access game is defined as $\mathcal{G}_{\mathbf{a}^{\prime}}=\left\{\mathcal{K},\left\{\mathcal{A}_{k}\right\}_{k \in \mathcal{K}},\left\{u_{k}\right\}_{k \in \mathcal{K}}\right\}$, where $\mathcal{K}$ is the player (SO) set, $\mathcal{A}_{k}$ is the action space of player $k$, and $u_{k}$ is the utility function of player $k$. The action space of each player is exactly the available channel set. To build a bridge between $\mathcal{G}_{\mathrm{a}^{\prime}}$ and problem (8), we give the definition of utility function of $k, k \in \mathcal{K}$ as follows.

$$
u_{k}=\Delta_{3, k} I_{k}(\mathbf{a})+\sum_{l \in \mathcal{K} \backslash\{k\}} \Delta_{3, l} p_{k} g_{k, b_{l}} \mathbb{1}_{\left\{a_{l}=a_{k}\right\}} .
$$

Then, we investigate the properties of $\mathcal{G}_{\mathbf{a}^{\prime}}$.

Theorem 1 If the variable $\mathbf{a}^{\prime}$ is predetermined and the potential function $\phi(\mathbf{a})=\sum_{l \in \mathcal{K}}\left(\Delta_{3, l} I_{l}(\mathbf{a})+\Delta_{4, l}\right), \mathcal{G}_{\mathbf{a}^{\prime}}$ is an exact potential game which exists at least one NE point $\mathbf{a}^{*}$. Moreover, the near-optimal solution to the proposed channel access problem (4) is a pure strategy $N E$ of $\mathcal{G}_{\mathbf{a}}$.

Proof The potential function of $\mathcal{G}_{\mathbf{a}^{\prime}}$ is defined as follows:

$$
\phi(\mathbf{a})=\sum_{l \in \mathcal{K}}\left(\Delta_{3, l} I_{l}(\mathbf{a})+\Delta_{4, l}\right) .
$$

Then, (10) is rewritten as (11).

$$
\begin{aligned}
\phi(\mathbf{a})= & \underbrace{\Delta_{3, k} I_{k}(\mathbf{a})+\sum_{l \in \mathcal{K} \backslash\{k\}} \Delta_{3, l} p_{k} g_{k, b_{l}} \mathbb{1}_{\left\{a_{l}=a_{k}\right\}}}_{u_{k}\left(a_{k}, \mathbf{a}_{-k}\right)} \\
& +\underbrace{\sum_{l \in \mathcal{K}}\left(\Delta_{3, l} \sigma_{l}^{2}+\Delta_{4, l}\right)+\sum_{l \in \mathcal{K} \backslash\{k\}}\left(\Delta_{3, l}\left(\sum_{m \in \mathcal{K} \backslash\{l, k\}} p_{m} g_{m, b_{l}} \mathbb{1}_{\left\{a_{m}=a_{l}\right\}}\right)\right)}_{v\left(\mathbf{a}_{-k}\right)}
\end{aligned}
$$

Suppose that an arbitrary player $k$ unilaterally changes its strategy from $a_{k}$ to $\bar{a}_{k}$, we can obtain the following equation based on (11):

$$
\phi\left(\bar{a}_{k}, \mathbf{a}_{-k}\right)-\phi\left(a_{k}, \mathbf{a}_{-k}\right)=u_{k}\left(\bar{a}_{k}, \mathbf{a}_{-k}\right)-u_{k}\left(a_{k}, \mathbf{a}_{-k}\right) .
$$

The equation above shows that the change in any single player's utility function due to unilateral strategy deviation results in exactly the same amount of change in the potential function. Therefore, according to Definition 2.2 in $[24,28], \mathcal{G}_{\mathbf{a}^{\prime}}$ is an exact potential game with potential function $\phi(\mathbf{a})$. As a kind of potential games, $\mathcal{G}_{\mathbf{a}^{\prime}}$ has some desirable properties, one of which is that $\mathcal{G}_{\mathbf{a}^{\prime}}$ exists at least one NE point.

Although each player in $\mathcal{G}_{\mathrm{a}^{\prime}}$ focuses on maximizing its own utility value, we characterize the achievable performance of NE points of $\mathcal{G}_{\mathrm{a}^{\prime}}$ by exploiting the inherent structure of the exact potential game.

Denote $\mathbf{a}^{\mathrm{opt}}$ as an optimal channel allocation profile that maximizes the potential function $\phi$, i.e.:

$$
\mathbf{a}^{\text {opt }} \in \arg \max _{\mathbf{a} \in \mathcal{A}} \phi(\mathbf{a}) .
$$

It has been proved that all NEs are the maximizers of the potential function $\phi$, either locally or globally, for any exact potential game [28]. The best equilibrium point is $\mathbf{a}^{\text {opt }}$. Obviously, $\mathbf{a}^{\text {opt }}$ is a near-optimal solution of (4) when $\mathbf{a}^{\prime}=\mathbf{a}^{*}$.

Hence, Theorem 1 is proved.

\section{Decentralized algorithm for achieving the best NE}

According to the above theoretic analysis of $\mathcal{G}_{\mathrm{a}^{\prime}}$, an approach of achieving the best NE of $\mathcal{G}_{\mathbf{a}^{\prime}}$ is also the approach to obtain a near-optimal solution of problem (4). In this section, we propose a distributed channel allocation learning algorithm to solve (4) in a distributed manner.

\subsection{Algorithm description}

Taking into account the above analysis, we give a detailed procedure of solving the channel allocation problem, labeled as Algorithm 1. Algorithm 1 includes two-tier loops of inner loop and outer loop, in which the variable $\mathbf{a}^{\prime}$ is updated until the near-optimal solution is achieved in inner loop. Two stages of inner loop in this improved algorithm are presented as follows: (a) In step 1, one player is randomly selected from the set of updatable players to update its strategy. Then, the selected player chooses an action and gets feedback in the form of the resulting state and an associated reward. (b) In step 2, the selected player updates its alternative action selection based on (14). In Algorithm 1, the stop criterion is set to be the case that the change of the potential function is trivial.

The proposed algorithm is not easily trapped in an undesirable NE when the game has multiple NE points because of its some favorable properties: (a) it is an uncoupled algorithm, namely, each player only needs to acquire the information of channel selection actions; (b) it can achieve the best NE which is the global optimum of maximizing potential function.

\subsection{Convergence and optimality analysis}

In order to investigate the actual performance of Algorithm 1, Theorems 2 and 3 characterize its convergence and optimality. 


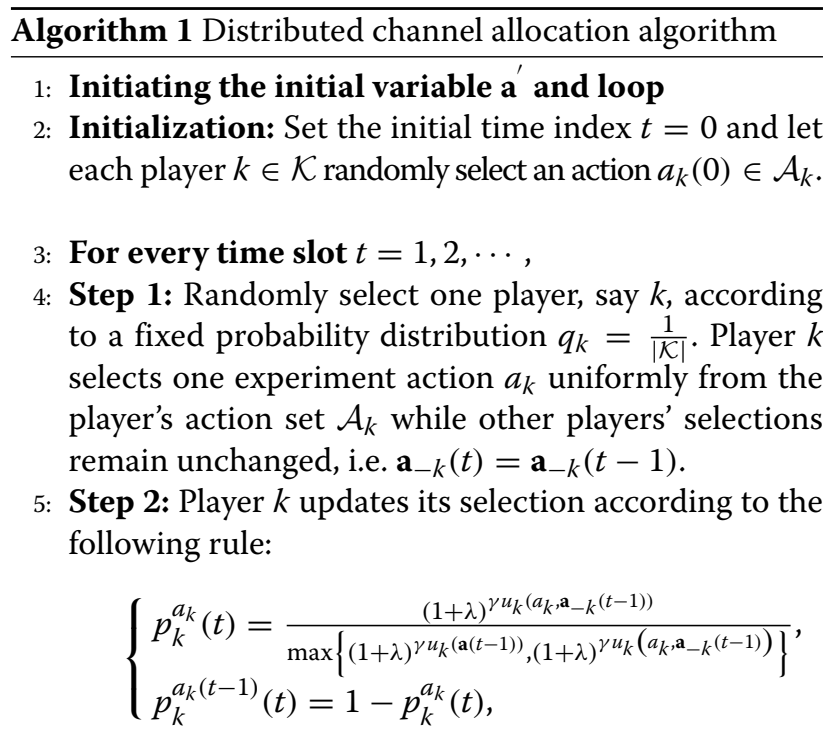

where $\lambda$ is the learning parameter and $\gamma$ is the smoothing factor. If the action $a_{k}$ is selected, let $\mathbf{a}^{*}(t)=\mathbf{a}(t)$; otherwise, $\mathbf{a}^{*}(t)=\mathbf{a}^{*}(t-1)$.

6: Step 3: If $\forall t$, there exists a transition probability $p_{k}(t)$ of player $k$, for $\forall k \in \mathcal{K}$, which is approaching one, e.g., larger than 0.99 , stop; otherwise, go to step 1.

7: If $\mathbf{a}^{\prime}=\mathbf{a}^{*}$, end loop; otherwise, return 2 and let $\mathbf{a}^{\prime}=\mathbf{a}^{*}$ if $\mathbf{a}^{*}$ is a better channel allocation strategy profile.

Theorem 2 If all players perform the proposed distributed channel allocation learning algorithm with the fixed a', the network converges to an unique stationary distribution of players' strategy profile, which is given by:

$$
\pi(\overline{\mathbf{a}})=\frac{(1+\lambda)^{\gamma \phi(\overline{\mathbf{a}})}}{\sum_{\mathbf{a} \in \mathcal{A}}(1+\lambda)^{\gamma \phi(\mathbf{a})}} .
$$

Proof Let $z(t)$ be the state of channel allocations at the $t$-th iteration of Algorithm 1 with the fixed a'. Obviously, $z(t)$ is an irreducible and aperiodic Markov process. Then, we will verify that the process determined by the distribution (15) is reversible. It is to say that for $\forall \mathbf{a}, \overline{\mathbf{a}} \in \mathcal{A}$, we have:

$$
\pi(\mathbf{a}) P(\overline{\mathbf{a}} \mid \mathbf{a})=\pi(\overline{\mathbf{a}}) P(\mathbf{a} \mid \overline{\mathbf{a}})
$$

where $P(\overline{\mathbf{a}} \mid \mathbf{a})$ is the the transition probability from state $\mathbf{a}$ to $\overline{\mathbf{a}}$.

When $\mathbf{a}=\overline{\mathbf{a}},(16)$ clearly holds. When $\mathbf{a} \neq \overline{\mathbf{a}}$, one player, say $k$, changes its working channel, which results in that one element of the network state has been changed, i.e., $\mathbf{a}=\left(a_{k}, \mathbf{a}_{-k}\right)$ and $\overline{\mathbf{a}}=\left(\bar{a}_{k}, \mathbf{a}_{-k}\right)$. It is easy for us to check that:

$$
\begin{aligned}
\pi(\mathbf{a}) P(\overline{\mathbf{a}} \mid \mathbf{a})= & \left(\frac{(1+\lambda)^{\gamma \phi(\mathbf{a})}}{\sum_{\tilde{\mathbf{a}} \in \mathcal{A}}(1+\lambda)^{\gamma \phi(\tilde{\mathbf{a}})}}\right)\left(\frac{1}{|\mathcal{K}|}\right) \\
& \left(\frac{(1+\lambda)^{\gamma u_{k}\left(\bar{a}_{k}, \mathbf{a}_{-k}\right)}}{\max \left\{(1+\lambda)^{\gamma u_{k}(\mathbf{a})},(1+\lambda)^{\gamma u_{k}\left(\bar{a}_{k}, \mathbf{a}_{-k}\right)}\right\}}\right) \\
= & c(1+\lambda)^{\gamma\left(\phi(\mathbf{a})+u_{k}\left(\bar{a}_{k}, \mathbf{a}_{-k}\right)\right)},
\end{aligned}
$$

where $c=c_{1} c_{2}, \quad c_{1}=\frac{1}{|\mathcal{K}| \sum_{\tilde{\mathbf{a}} \in \mathcal{A}}(1+\lambda)^{\gamma \phi(\tilde{\mathbf{a}})}}$, and $c_{2}=$ $\frac{1}{\max \left\{(1+\lambda)^{\gamma u_{k}(\mathbf{a})},(1+\lambda)^{\gamma u_{k}\left(\bar{a}_{k}, \mathbf{a}-k\right)}\right\}}$.

According to the symmetry, we have:

$$
\pi(\overline{\mathbf{a}}) P(\mathbf{a} \mid \overline{\mathbf{a}})=c(1+\lambda)^{\gamma\left(\phi(\overline{\mathbf{a}})+u_{k}\left(a_{k}, \mathbf{a}_{-k}\right)\right)} .
$$

By substituting (12) into (17), we can obtain:

$$
\begin{aligned}
\pi(\mathbf{a}) P(\overline{\mathbf{a}} \mid \mathbf{a}) & =c(1+\lambda)^{\gamma\left(\phi(\overline{\mathbf{a}})+u_{k}\left(a_{k}, \mathbf{a}_{-k}\right)\right)} \\
& =\pi(\overline{\mathbf{a}}) P(\mathbf{a} \mid \overline{\mathbf{a}}) .
\end{aligned}
$$

Thus, we can derive that:

$$
\sum_{\mathbf{a} \in \mathcal{A}} \pi(\mathbf{a}) P(\overline{\mathbf{a}} \mid \mathbf{a})=\sum_{\mathbf{a} \in \mathcal{A}} \pi(\overline{\mathbf{a}}) P(\mathbf{a} \mid \overline{\mathbf{a}})=\pi(\overline{\mathbf{a}}),
$$

which is the balanced equation of Markov process.

Hence, Theorem 2 is proved.

Theorem 3 If the variable a' is fixed, the inner loop of Algorithm 1 converges to the best $N E$ point of $\mathcal{G}_{\mathbf{a}^{\prime}}$ with an arbitrarily high probability when $\gamma$ is sufficiently large. Therefore, the MOS level of the IoT network is approximately maximized when a is the NE point.

Proof It is noted from Theorem 1 that $\mathbf{a}^{\text {opt }}$ is represented as an optimal channel allocation profile that maximizes the potential function $\phi$, which is also the best NE of $\mathcal{G}_{\mathbf{a}^{\prime}}$.

According to Theorem 2, the proposed algorithm converges to a unique stationary distribution. When $\gamma$ is sufficiently large, $(1+\lambda)^{\gamma \phi\left(\mathbf{a}^{\mathrm{opt}}\right)} \gg(1+\lambda)^{\gamma \phi(\mathbf{a})}, \forall \mathbf{a} \in \mathcal{A} \backslash$ $\{\mathbf{a}\}$. According to (15), the unique stationary distribution of players' strategy profile is $(0, \ldots, 0,1,0, \ldots, 0)$, where 1 denotes the probability of the optimal channel allocation solution and the probabilities of other non-optimal solutions are all 0 . Thus:

$$
\lim _{\gamma \rightarrow \infty} \pi\left(\mathbf{a}^{\mathrm{opt}}\right)=1
$$

which means that the proposed learning algorithm converges to the best NE of $\mathcal{G}_{\mathbf{a}^{\prime}}$ with an arbitrarily high probability. When $\mathbf{a}^{\prime}=\mathbf{a}^{\text {opt }}$, the NE obtained by the proposed algorithm is a near-optimum solution to problem (8). Then, the MOS level of the IoT network is approximately maximized when $\mathbf{a}^{\prime}$ is the NE point.

Thus, the proof is completed. 


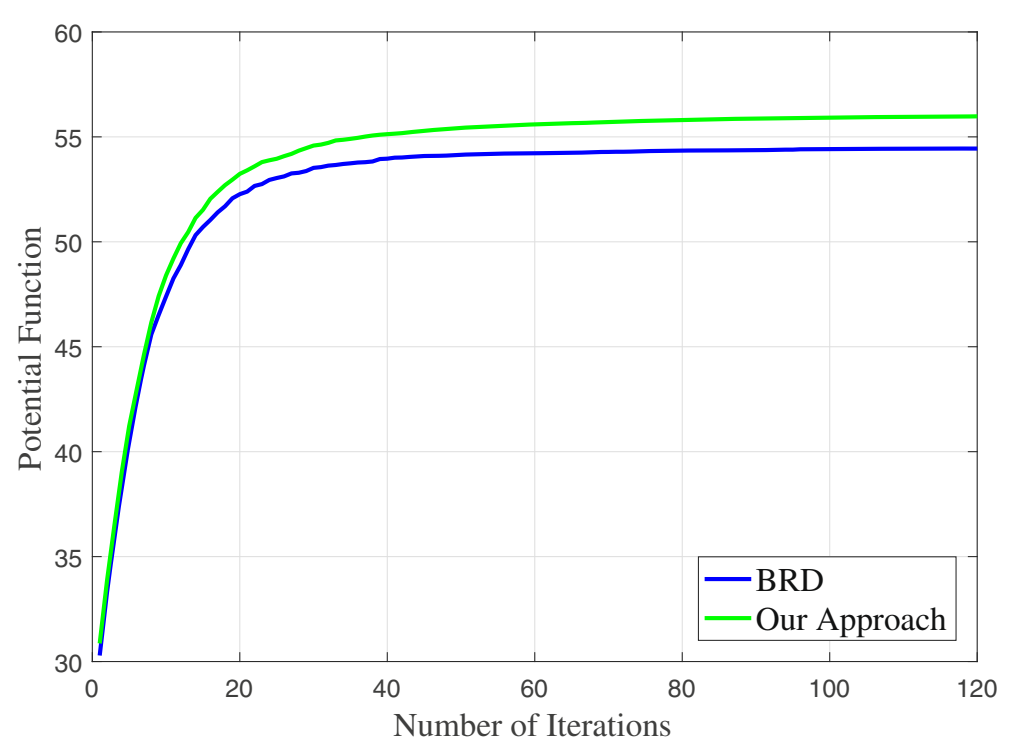

Fig. 3 The comparison results of the convergence performance of Algorithm 1 and BRD with fixed $\mathbf{a}$

\section{Simulation results and discussion}

In this section, numerical simulations are performed by Matlab software to validate the efficiency and performance of our proposed algorithm for solving the channel allocation problem of IoT uplink communications over cellular networks.

\subsection{Scenario setup}

We consider one MBS with a hexagonal coverage area where there are randomly layouts of 2 SCBSs. For convenience, we assume that there are 3 SOs randomly located in each SCBS and the other 10 SOs in the coverage area of MBS. Here, we suppose that each SO has the same uplink transmission power which is set to $23 \mathrm{dBm}$. Accordingly, suppose that the total $5 \mathrm{MHz}$ bandwidth in this heterogeneous network constitutes $N=10$ channels with each same bandwidth $487.5 \mathrm{kHz}$. Each SO chooses 1 channel for transmission. Rayleigh fading model is considered in the simulation and $h_{b_{c}}$ is the link gain from SO $d$ to BS $b_{c}$, which is expressed as $h_{b_{c}}=\xi_{b_{c}}\left(L_{b_{c}}\right)^{-\theta}$, where $L_{b_{c}}$ is the distance between SO $d$ and BS $b_{c}, \xi_{b_{c}}$ denotes the channel fading component and $\theta$ is the path loss exponent. The
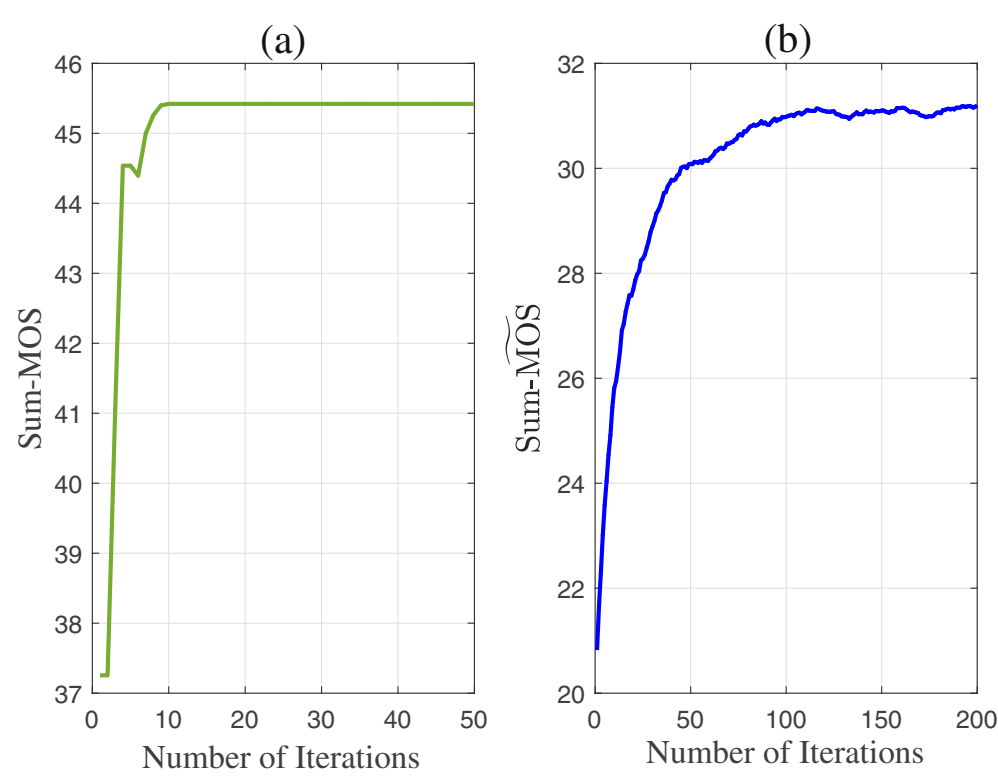

Fig. 4 The changing curves of the optimization objectives as the number of iterations increases by Algorithm 1 with fixed $\mathbf{a}^{\prime}: \mathbf{a}$ in problem $P ; \mathbf{b}$ in problem $\tilde{P}$ 


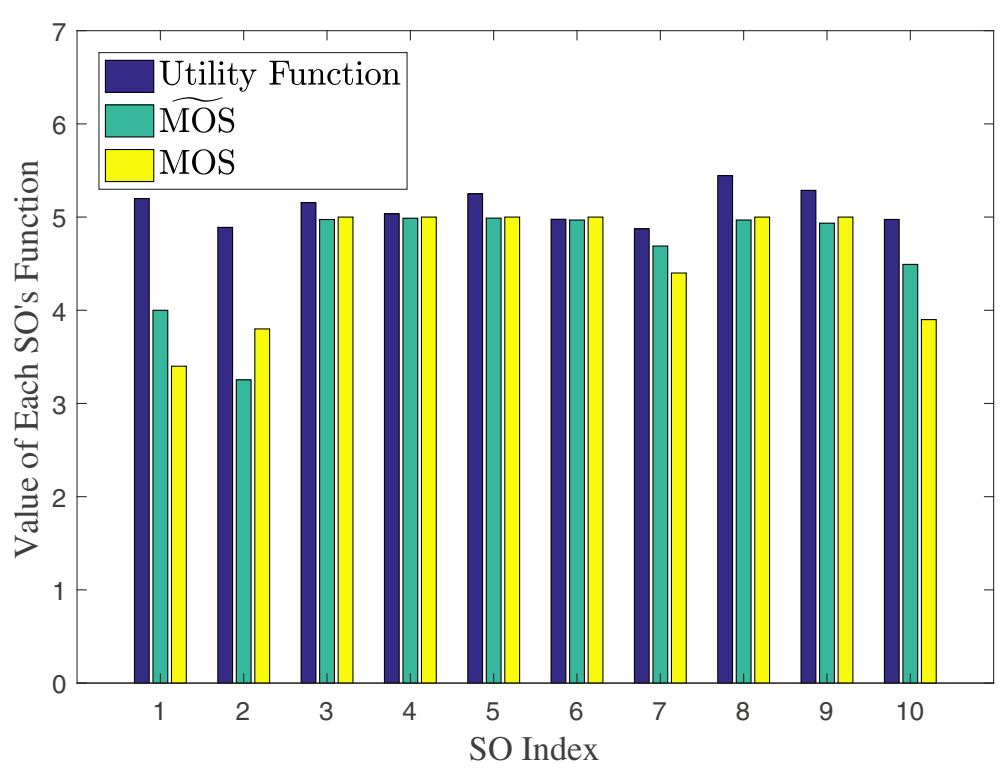

Fig. 5 The comparisons of utility, $\widetilde{M O S}$ and MOS for each SO obtained by preforming Algorithm 1

noise power is set to $\sigma^{2}=-174 \mathrm{dBm} / \mathrm{Hz}$. In the following simulations, the simulation results are obtained by 400 independent trials and those parameters involved are optimized by experiments.

\subsection{Convergence behavior and optimality of this algorithm}

In this subsection, we first investigate the convergence behavior comparison between Algorithm 1 and best response dynamic (BRD). It is shown from Fig. 3 that Algorithm 1 and BRD can respectively converge to two stable points as the number of iterations increases. Compared with BRD, Algorithm 1 has a faster convergence speed and achieves a better solution. It is supported with the proved fact that BRD can only converge to one NE of the potential game which may be not the best NE. Conversely, our algorithm can find the the best NE which is also the optimal solution of maximizing potential function. Therefore, the proposed algorithm is distributed and can obtain a better convergence performance. Figure 4 plots the changing curves of the optimization objectives in problems $P$ and $\tilde{P}$ as the number of iterations increases by performing Algorithm 1 with fixed $\mathbf{a}^{\prime}$. It is shown from Fig. 4 that sum-MOS in problem $P$ gradually increases and converges eventually along with the increase of iteration times, which is consistent with the variation tendency of

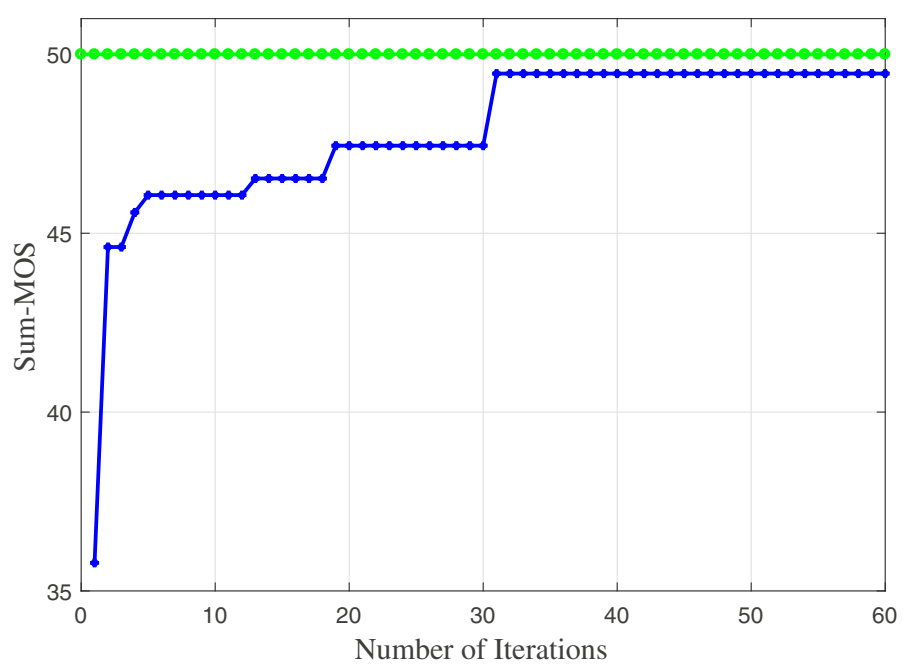

Fig. 6 The changing curve of sum-MOS as the number of iterations increases by preforming Algorithm 1 
sum- $\widetilde{M O S}$ in problem $\tilde{P}$. This indicates that the increase of sum- $\widetilde{\mathrm{MOS}}$ by selecting better channel allocation strategy profile also causes the improvement of sum-MOS. Although sum- $\widetilde{\mathrm{MOS}}$ continues to increase in the latter process, the value of sum-MOS is unchangeable. Since MOS is a piecewise function, the better solution for $\tilde{P}$ cannot further enhance the performance of $P$, which implies that multiple optimal solutions exist.

In the following, we evaluate the MOS performance of each SO by preforming Algorithm 1. From Fig. 5, it is worth noting that Algorithm 1 can maintain a better SO fairness with respect to MOS performance by taking into account the impact of the interference generated by each $\mathrm{SO}$ on the entire network. Algorithm 1 is proposed to find the best $\mathrm{NE}$ of the channel access game $\mathcal{G}_{\mathbf{a}^{\prime}}$, which achieves an approximately equal utility value for each $\mathrm{SO}$ shown in Fig. 5. Moreover, the fairness among SOs with respect to MOS or $\widetilde{M O S}$ performance is guaranteed. Figure 6 plots the changing curve of sum-MOS as the number of iterations increases by preforming Algorithm 1. It is shown that Algorithm 1 can improve the QoE of SOs. However, our proposed algorithm cannot guarantee convergence to the global optimal solution of $\tilde{P}$ since the potential function in $\mathcal{G}_{\mathbf{a}^{\prime}}$ is different from the optimization objective in $\tilde{P}$ where $\widetilde{\mathrm{MOS}}$ is a piecewise function. The best NE $\mathbf{a}^{*}$ of $\mathcal{G}_{\mathbf{a}^{*}}$, i.e., the maximum of potential function, is obtained by performing Algorithm 1 which is only the near-optimum of $P$. It is noted from Fig. 6 that sum-MOS in $P$ can eventually converge to a fixed point as the number of iterations increases and is close to the maximum value of sum-MOS. This indicates that our approach provides high performance for solving this difficult non-convex optimization problem.

\section{Conclusion}

In this paper, we investigated the channel allocation problem in 5G-enabled IoT, by using a game-theoretic learning algorithm, to improve the QoE of SOs. In order to measure the QoE of SOs in IoT, we first defined a MOS function. Then, we proposed the exact potential game to formulate this optimization problem, in which the potential function was designed by approximatively converting the objective function into a tractable form. It was proved that the exact potential game existed the best $\mathrm{NE}$ which was a near optimization solution of the channel allocation problem. Aiming at the proposed game, we designed a distributed learning algorithm and proven it can converge to the best NE with an arbitrarily high probability.

\section{Abbreviations}

BRD: Best response dynamic; loT: Internet of things; MBS: Macrocell base station; MOS: Mean opinion score; NE: Nash equilibrium; QoE: Quality of experience; SCBSs: Small cell base stations; SINR:

Signal-to-interference-plus-noise ratio; SOs: Smart objects

\section{Funding}

This work was supported by the National Natural Science Foundation of China (Grant No. 61801243), by the Natural Science Foundation of the Jiangsu Higher Education Institutions of China (Grant No. 18KJB510026), and by the Foundation of Nanjing University of Posts and Telecommunications (Grant No. NY218124).

\section{Availability of data and materials}

Mostly, I got the writing material from different journals as presented in the references. A MATLAB tool has been used to simulate my concept.

\section{Authors' contributions}

All authors contributed significantly to the research work presented in this paper. HD had a leading role in the formulation and solution of the considered optimization problem, while performing a detailed evaluation and analysis of the developed algorithm, through conducting an extensive set of simulations. $\mathrm{HD}$ and $\mathrm{HZ}$ completed the writing and formatting of the paper. WW did the experiments and simulations. BW helped in finalizing the solution and amending the manuscript. All authors read and approved the final manuscript.

\section{Competing interests}

The authors declare that they have no competing interests.

\section{Publisher's Note}

Springer Nature remains neutral with regard to jurisdictional claims in published maps and institutional affiliations.

\section{Author details}

${ }^{1}$ School of Internet of Things, Nanjing University of Posts and

Telecommunications, 66 Xin mofan Road, 210003 Nanjing, China. 2Engineering Systems and Design Pillar, Singapore University of Technology and Design, 8 Somapah Road, 487372 Singapore, Singapore. ${ }^{3}$ College of Telecommunications and Information Engineering, Nanjing University of Posts and Telecommunications, 66 Xin mofan Road, 210003 Nanjing, China.

Received: 15 June 2018 Accepted: 29 January 2019

Published online: 06 March 2019

References

1. M. A. M. Albreem, A. A. El-Saleh, M. Isa, W. Salah, M. Jusoh, M. M. Azizan, A. Ali, in 2017 IEEE 4th International Conference on Smart Instrumentation, Measurement and Application (ICSIMA). Green Internet of things (IoT): an overview, (2017), pp. 1-6

2. W. Tan, M. Matthaiou, S. Jin, X. Li, Spectral efficiency of dft-based processing hybrid architectures in massive mimo. IEEE Wirel. Commun. Lett. 6(5), 586-589 (2017)

3. A. Musaddiq, Y. B. Zikria, O. Hahm, H. Yu, A. K. Bashir, S. W. Kim, A survey on resource management in loT operating systems. IEEE Access. 6 , 8459-8482 (2018)

4. W. Tan, S. Jin, C.-K. Wen, T. Jiang, Spectral efficiency of multi-user millimeter wave systems under single path with uniform rectangular arrays. EURASIP Wirel, J., Commun. Netw. 2017(1), 181 (2017)

5. X. Sun, N. Ansari, Dynamic resource caching in the loT application layer for smart cities. IEEE Internet Things J. 5(2), 606-613 (2018)

6. Z. Qin, J. A. McCann, in 2017 IEEE Global Communications Conference. Resource efficiency in low-power wide-area networks for loT applications, (2017), pp. 1-7

7. B. Khalfi, B. Hamdaoui, M. Guizani, Extracting and exploiting inherent sparsity for efficient loT support in 5G: Challenges and potential solutions. IEEE Wirel. Commun. 24(5), 68-73 (2017)

8. C. Li, Y. Li, K. Song, L. Yang, Energy efficient design for multiuser downlink energy and uplink information transfer in 5G. Sci. China Inf. Sci. 59(2), 1-8 (2016)

9. J. Yuan, S. Jin, W. Xu, W. Tan, M. Matthaiou, K. K. Wong, User-centric networking for dense C-RANs: high-SNR capacity analysis and antenna selection. IEEE Trans. Commun. 65(11), 5067-5080 (2017)

10. M. Zhang, W. Tan, J. Gao, S. Jin, Spectral efficiency and power allocation for mixed-ADC massive MIMO system. China Commun. 15(3), 112-127 (2018) 
11. W. Tan, D. Xie, J. Xia, W. Tan, L. Fan, S. Jin, Spectral and energy efficiency of massive MIMO for hybrid architectures based on phase shifters. IEEE Access. 6, 11751-11759 (2018)

12. H. Ji, S. Park, J. Yeo, Introduction to ultra reliable and low latency communications in 5G (2017). arXiv preprint arXiv:1704.05565

13. C. Li, K. Song, D. Wang, F. Zheng, L. Yang, Optimal remote radio head selection for cloud radio access networks. Sci. China Inf. Sci. 59(10), 102315:1-102315:12 (2016)

14. S. Li, Q. Ni, Y. Sun, G. Min, S. Al-Rubaye, Energy-efficient resource allocation for industrial cyber-physical loT systems in $5 \mathrm{G}$ era. IEEE Trans. Ind. Inform. 4(6), 2618- 28 (2018)

15. C. Li, S. Zhang, P. Liu, F. Sun, J. M. Cioffi, L. Yang, Overhearing protocol design exploiting intercell interference in cooperative green networks. IEEE Trans. Veh. Technol. 65(1), 441-446 (2016)

16. H. Dai, Y. Huang, J. Wang, L. Yang, Resource optimization in heterogeneous cloud radio access networks. IEEE Commun. Lett. 22(3), 494-497 (2018)

17. A. K. Yerrapragada, B. Kelley, in 2017 12th System of Systems Engineering Conference (SOSE). An loT self organizing network for $5 \mathrm{G}$ dense network interference alignment, (2017), pp. 1-6

18. N. N. Dao, M. Park, J. Kim, Resource-aware relay selection for inter-cell interference avoidance in $5 \mathrm{G}$ heterogeneous network for internet of things systems. Futur. Gener. Comput. Syst. (2018)

19. W. Ejaz, M. Ibnkahla, Multiband spectrum sensing and resource allocation for loT in cognitive 5G networks. IEEE Internet Things J. 5(1), 150-163 (2018)

20. A. Aminjavaheri, A. RezazadehReyhani, R. Khalona, Underlay control signaling for ultra-reliable low-latency loT communications (2018). arXiv preprint arXiv:1711.02248

21. X. He, K. Wang, H. Huang, T. Miyazaki, Y. Wang, S. Guo, Green resource allocation based on deep reinforcement learning in content-centric loT. IEEE Trans. Emerg. Top. Comput. 9(3), 1-15 (2018)

22. M. Aazam, M. St-Hilaire, C. H. Lung, I. Lambadaris, in 201623 rd International Conference on Telecommunications (ICT). MeFoRE: QoE based resource estimation at Fog to enhance QoS in loT, (2016), pp. 1-5

23. J. Huang, Y. Yin, Q. Duan, H. Yan, in 2015 3rd International Conference on Future Internet of Things and Cloud. A game-theoretic analysis on context-aware resource allocation for device-to-device communications in cloud-centric internet of things, (2015), pp. 80-86

24. H. Dai, Y. Huang, R. Zhao, J. Wang, L. Yang, Resource optimization for device-to-device and small cell uplink communications underlaying cellular networks. IEEE Trans. Veh. Technol. 67(2), 1187-1201 (2018)

25. H. Zhang, Y. Xiao, S. Bu, D. Niyato, F. R. Yu, Z. Han, Computing resource allocation in three-tier loT Fog networks: a joint optimization approach combining stackelberg game and matching. IEEE Internet Things J. 4(5), 1204-1215 (2017)

26. S. F. Abedin, M. G. R. Alam, N. H. Tran, C. S. Hong, in 2015 17th Asia-Pacific Network Operations and Management Symposium APNOMS). A Fog based system model for cooperative loT node pairing using matching theory, (2015), pp. 309-314

27. A. Rullo, D. Midi, E. Serra, E. Bertino, in 2016 IEEE 36th International Conference on Distributed Computing Systems (ICDCS). Strategic security resource allocation for internet of things, (2016), pp. 737-738

28. H. Dai, Y. Huang, C. Li, S. Li, L. Yang, Energy-efficient resource allocation for device-to-device communication with WPT. IET Commun. 11(3), 326-334 (2017)

\section{Submit your manuscript to a SpringerOpen ${ }^{\circ}$ journal and benefit from:}

- Convenient online submission

Rigorous peer review

- Open access: articles freely available online

- High visibility within the field

- Retaining the copyright to your article

Submit your next manuscript at $\gg$ springeropen.com 